\title{
A Novel Electrochemical Sensor Based on the Synergistic Effect of Trace Platinum and Foliate $\mathrm{Co}_{0.85}$ Se for the Determination of Dopamine
}

\author{
Xiang Li, ${ }^{1,2}$, Miaomiao Chen ${ }^{1,2}$, Rong Rui ${ }^{1}$, Zhigui Wan ${ }^{1}$, Feng Chen ${ }^{1}$, Xiaoshuang Zuo ${ }^{1}$, \\ Chang Wang $^{1 *}$, Hai $\mathrm{Wu}^{1,2 *}$ \\ ${ }^{1}$ School of Chemistry and Materials Engineering, FuyangNormalUniversity, Fuyang, Anhui 236037, \\ PR China \\ ${ }^{2}$ Anhui Province Key Laboratory of Environmental Hormone and Reproduction, Anhui Provincial \\ Key Laboratory for Degradation and Monitoring of Pollution of the Environment, Fuyang Normal \\ University. Fuyang, Anhui 236037, PR China. \\ *E-mail: bigceleron@163.com; wuhai317@126.com
}

doi: $10.20964 / 2019.05 .68$

Received: 12 January 2019 / Accepted: 19 March 2019 / Published: 10 April 2019

Foliate cobalt selenide $\left(\mathrm{Co}_{0.85} \mathrm{Se}\right)$ was synthesized by the hydrothermal method and was combined with trace platinum $(\mathrm{Pt})$ to form a $\mathrm{Pt} / \mathrm{Co}_{0.85} \mathrm{Se}$ material, which was modified on the surface of a glassy carbon electrode (GCE) to fabricate a novel nonenzymatic electrochemical sensor ( $\mathrm{Pt} / \mathrm{Co}_{0.85} \mathrm{Se} / \mathrm{GCE}$ ) for the selective determination of dopamine (DA). On the $\mathrm{Pt} / \mathrm{Co}_{0.85} \mathrm{Se}$ interface, well-separated oxidation peaks of DA, ascorbic acid (AA), and uric acid (UA) were observed due to the superior conductivity of $\mathrm{Co}_{0.85} \mathrm{Se}$ and the excellent catalytic activity of Pt. With the synergistic effect of Pt and $\mathrm{Co}_{0.85} \mathrm{Se}$, the linear range for DA detection was found to be $0.50-22.0 \mu \mathrm{M}$ with a detection limit of $0.39 \mu \mathrm{M}(\mathrm{S} / \mathrm{N}=3)$ and a high sensitivity of $2306 \mu \mathrm{A} \mathrm{mM} \mathrm{mm}^{-1} \mathrm{~cm}^{-2}$. Furthermore, the proposed sensor exhibited high selectivity for the determination of DA in the presence of UA, AA, and other potential interferences, which indicated that the sensor showed great potential for practical and reliable DA analysis of serum samples.

Keywords: Electrochemical sensor, Cobalt selenide, Dopamine, Platinum

\section{FULL TEXT}

(C) 2019 The Authors. Published by ESG (www.electrochemsci.org). This article is an open access article distributed under the terms and conditions of the Creative Commons Attribution license (http://creativecommons.org/licenses/by/4.0/). 